\title{
Stem cell jackpot for Parkinson's disease
}

\section{By Lev Osherovich, Senior Writer}

A Memorial Sloan-Kettering Cancer Center team has honed a protocol for producing large quantities of human dopaminergic neurons that could be used therapeutically by grafting them into patients with Parkinson's disease or as a platform for drug screening for the disease. ${ }^{1}$ But scaling up the protocol remains a challenge, and therapeutic applications face major regulatory issues due to potential safety concerns.

Parkinson's disease (PD) is caused by degeneration of dopaminergic neurons throughout the brain, the effects of which are felt most acutely in the substantia nigra, a midbrain region involved in movement.

"The idea of stem cells for PD is not new, but we've never had a good source of enriched dopaminergic cells for transplantation."

-Lorenz Studer,

Memorial Sloan-Kettering Cancer Center
PD symptoms are treated with L-dopa, a dopamine precursor, but due to side effects, limited efficacy and the inconvenience of frequent dosing with this compound or other dopamine agonists, research has turned to finding a way to restore dopamine levels using neuronal implants.

Since the late $1980 \mathrm{~s}$, researchers have tried to replace dying neurons of the substantia nigra with dopaminergic cell grafts from aborted fetuses. But despite longterm engraftment and modest clinical efficacy, the limited availability of source material means that the approach cannot be scaled up.

Thus, "there has been an effort to increase the yield of dopaminergic cells in cell culture prior to transplantation," said Curt Freed, division head and professor of medicine at the University of Colorado Denver School of Medicine, who conducted the first fetal cell grafts in PD patients.

Human embryonic stem cells (ESCs), which can be cultured indefinitely, are potentially a scalable alternative to primary fetal tissue. However, obtaining high yields of stable dopaminergic cells without also producing unwanted nondopaminergic cells has been a challenge.

Now, a team led by Lorenz Studer, professor of developmental biology and director of the Center for Stem Cell Biology at Sloan-Kettering, has optimized an ESC culture procedure that yields large quantities of precisely the kind of dopaminergic cells needed to treat PD.

"The idea of stem cells for PD is not new, but we've never had a good source of enriched dopaminergic cells for transplantation," said Studer.

\section{Cells well}

Studer got a hint of how to obtain the dopaminergic cells from his team's earlier efforts to coax ESCs into forming various neuronal precursors. In one of those prior studies, the team identified markers for midbrain dopaminergic neuron precursors and developed a procedure to grow a specialized subset of those cells, called floor plate precursors, in vitro. ${ }^{2}$

In the new study, Studer's team converted the floor plate cells into functioning midbrain neurons by simultaneously manipulating several signaling pathways that influence neuronal development.

"The way to go from stem cells to highly specialized nerve cells is to give a series of instructions for differentiation," said Studer. The key signal to make the right kind of neurons turned out to be activation of the wingless-type MMTV integration site (WNT) signaling pathway.

Studer's technique involves treating floor plate precursors with a trio of compounds-a small molecule inhibitor of glycogen synthase kinase $3 \beta$ (GSK3B) that activates the WNT signaling pathway, a sonic hedgehog homolog ( $\mathrm{SHH}$ ) agonist and recombinant fibroblast growth factor 8 (FGF8). ESCs treated with all three agents showed high levels of midbrain dopaminergic neuron markers compared with cells treated only with the SHH agonist and FGF8.

In cell culture, the ESC-derived neurons behaved like natural dopaminergic neurons, secreting more dopamine and lower amounts of other neurotransmitters like serotonin and $\gamma$-aminobutyric acid (GABA) than neurons generated by previous in vitro methods. Immunohistochemical analysis showed that Studer's method also produced more dopaminergic neurons than other types of neurons.

Next, Studer's team transplanted the in vitro-generated dopaminergic neurons into mouse, rat and monkey models of PD and found that the neurons successfully engrafted, survived indefinitely and restored dopaminergic activity in the midbrain. PD animals receiving Studer's dopaminergic neuron grafts had higher midbrain dopaminergic neuron density and better performance in gait assays than animals given dopaminergic neurons made by prior methods.

Studer's dopaminergic neuron grafts appear to be stable over the long term. Mice receiving these cells stably expressed dopaminergic cell markers, showed no signs of contaminating cell overgrowth and showed improved performance in an assay of amphetamine-induced motion disorder as late as 16 weeks after transplantation compared with controls receiving neuronal preparations made by prior methods.

\section{Raising yield}

Academic experts polled by $S c i B X$ said that from a technical standpoint, Studer's new method is an incremental advance over previous methods, but the resulting increase in efficiency is potentially a game changer for manufacturing dopaminergic cells.

"The novelty of the report is the accomplishment of a differentiation protocol that more reliably generates the correct cell type," said Ole Isacson, professor of neuroscience and neurology at Harvard Medical School. "This is definitely an improvement on prior protocols reported by the same group a few years ago."

Isacson noted that his own team recently reported that transplantation of mouse ESC-derived midbrain dopaminergic neurons 
isolated by fluorescence-activated cell sorting (FACS) can ameliorate a rat model of PD. ${ }^{3}$ Studer's method provides a potential source of large numbers of such neurons without the need for time-consuming and inefficient FACS protocols.

Although about $80 \%$ of the cells in Studer's in vitro preparation are dopaminergic neurons, it is not clear what fraction of the starting ESCs are converted into neurons by Studer's complex procedure.

"The next challenge is to achieve sufficient yield," said Isacson. "It's not clear whether they've generated more neurons overall or a higher percentage of the right neurons."

Studer's method could solve several challenges to ESC therapies for PD, including concerns about graft purity and the potential for tumor formation.

Freed and Isacson said that previous ESC culture methods ran the risk of generating unwanted cells, in particular serotonergic neurons, which can interfere with the activity of dopaminergic cells. In contrast, Studer's team showed that neurons cultured according to the new protocol did not secrete serotonin. Indeed, at 4.5 months after transplantation, mice receiving grafts grown by Studer's new method had very few transplanted cells with markers of nondopaminergic cell identity compared with mice receiving conventional neural grafts.

Freed said that for regulators to accept the cells as therapeutic candidates, long-term preclinical safety studies would be needed to exclude the possibility of tumor formation. Freed said that in contrast to his original fetal cell graft studies, which at the time did not fall under FDA regulation, today the regulatory environment for cell therapies is much more stringent.

"A renegade stem cell is a potential disaster," said Freed. "FDA might say that because Studer didn't report much data about tumors, they would like to see 100 mice for a year who have no tumors with this preparation."

"We now have right cells," said Studer. "The question is now how to make these cells in a format that's safe to use" in the clinic. Doing so would require making the cells under GMP conditions, which would likely require collaboration with a cell manufacturing company, he added.

\section{Cell side}

Cell therapies for PD have not made much headway since the cessation of fetal transplant studies in the 1990s. NeuroGeneration Inc.'s Phase II trial of its neural stem cell-derived dopaminergic cell therapy has been on hold since 2008 due to cell manufacturing concerns. Last month, Geron Corp. discontinued its ESC therapy program, which included a Phase I trial of oligodendrocyte progenitor cells in acute spinal cord injury (SCI).

Two companies-BrainStorm Cell Therapeutics Inc. and International Stem Cell Corp.- - have preclinical programs to generate stem cell-derived therapies for PD. Those approaches use mesenchymal and parthenogenetically derived stem cells, respectively, but these cell types are not thought to be as readily programmable into specific neurons as ESCs.

Isacson, Freed and Studer all noted that the complexity of the regulatory path and pessimism about stem cell therapies mean that, in the short term, the likeliest commercial use for the new dopaminergic cells would be in in vitro drug screening assays.

"Because these cells are much closer to the real dopaminergic cells [than previous cells], they are suitable for drug screening," said Studer.

Cellular reagent company Cellular Dynamics International Inc. is negotiating a license to Studer's technology. Chris Parker, VP and chief commercial officer of CDI, noted that Studer's cells would fit well with the company's portfolio of specialized neuronal cells for drug screening.

Parker said that to be commercially useful, Studer's protocol would need to be scaled up at least 1,000-fold, but such scaling often requires major changes to the cell culture protocols.

"In this paper, they make millions of cells per animal, but we would have to make billions and billions of cells for this to be useful as a screening platform," said Parker.

Scaling up the procedure will require considerable refinement.

"If you're going to make a product, you have to know how many of these cells will survive freezing and thawing, how many will stick to a matrix, how many of them form the appropriate cell type," said Parker. "For every cell line, the protocol must be optimized and modified."

Studer said his next step is to scale up production of his dopaminergic neurons.

He has filed a patent on his methods, which is available for licensing.

Osherovich, L. SciBX 4(46); doi:10.1038/scibx.2011.1285

Published online Dec. 1, 2011

\section{REFERENCES}

1. Kriks, S. et al. Nature; published online Nov. 6, 2011; doi:10.1038/nature10648

Contact: Lorenz Studer, Memorial Sloan-Kettering Cancer Center, New York, N.Y. e-mail: studerl@mskcc.org

2. Chambers, S.M. et al. Nat. Biotechnol. 27, 275-280 (2009)

3. Hedlund, E. et al. Stem Cells 26,1526-1536 (2008)

COMPANIES AND INSTITUTIONS MENTIONED

BrainStorm Cell Therapeutics Inc. (OTCQB:BCLI), New York, N.Y. Cellular Dynamics International Inc., Madison, Wis.

Geron Corp. (NASDAQ:GERN), Menlo Park, Calif.

Harvard Medical School, Boston, Mass.

International Stem Cell Corp. (OTCBB:ISCO), Carlsbad, Calif.

Memorial Sloan-Kettering Cancer Center, New York, N.Y.

NeuroGeneration Inc., Los Angeles, Calif.

University of Colorado Denver School of Medicine, Aurora, Colo. 